\title{
ALGEBRAS OF ITERATED PATH INTEGRALS AND FUNDAMENTAL GROUPS $\left(^{1}\right)$
}

\author{
BY \\ KUO-TSAI CHEN
}

\begin{abstract}
A method of iterated integration along paths is used to extend deRham cohomology theory to a homotopy theory on the fundamental group level. For every connected $C^{\infty}$ manifold $\mathfrak{M}$ with a base point $p$, we construct an algebra $\pi^{1}=\pi^{1}(\mathfrak{M}, p)$ consisting of iterated integrals, whose value along each loop at $p$ depends only on the homotopy class of the loop. Thus $\pi^{1}$ can be taken as a commutative algebra of functions on the fundamental group $\pi_{1}(\mathfrak{M})$, whose multiplication induces a comultiplication $\pi^{1} \rightarrow \pi^{1} \otimes \pi^{1}$, which makes $\pi^{1}$ a Hopf algebra. The algebra $\pi^{1}$ relates the fundamental group to analysis of the manifold, and we obtain some analytical conditions which are sufficient to make the fundamental group nonabelian or nonsolvable. We also show that $\pi^{1}$ depends essentially only on the differentiable homotopy type of the manifold.

The second half of the paper is devoted to the study of structures of algebras of iterated path integrals. We prove that such algebras can be constructed algebraically from the following data: (a) the commutative algebra $A$ of $C^{\infty}$ functions on $\mathfrak{M}$; (b) the $A$-module $M$ of $C^{\infty} 1$-forms on $\mathfrak{M}$; (c) the usual differentiation $d: A \rightarrow M$; and (d) the evaluation map at the base point $p, \varepsilon: A \rightarrow K, K$ being the real (or complex) number field.
\end{abstract}

A path $\alpha:[0,1] \rightarrow \mathfrak{M}$ will be understood to be piecewise smooth. For $w, w_{1}, w_{2}, \cdots \in M$, let $\int_{\alpha} w$ be the usual integral, and define, for $r>1$,

$$
\int_{\alpha} w_{1} \cdots w_{r}=\int_{0}^{1}\left(\int_{\alpha^{t}} w_{1} \cdots w_{r-1}\right) w_{r}(\alpha(t), \dot{\alpha}(t)) d t,
$$

where $\alpha^{t}$ denotes the restriction $\alpha \mid[0, t]$.

Such iterated path integrals can be taken as functions on a space of paths. The integral $\int w_{1} \cdots w_{r}$, taken to be a $K$-valued function on the set of paths (resp. loops) from the base point $p$, will be written as $\int_{p} w_{1} \cdots w_{r}$ (resp. $\left.\oint_{p} w_{1} \cdots w_{r}\right)$. The totality of such path integrals $\int_{p} w_{1} \cdots w_{r}$ (resp. $\left.\oint_{p} w_{1} \cdots w_{r}\right), r \geqq 1$, together with the constant function 1 generate an algebra $\boldsymbol{P}$ (resp. $\boldsymbol{Q}$ ). Actually $\boldsymbol{Q}$ is a Hopf algebra with a comultiplication $\zeta_{\boldsymbol{Q}}: \boldsymbol{Q} \rightarrow \boldsymbol{Q} \otimes \boldsymbol{Q}$ reflecting the multiplication of loops.

Those elements of $\boldsymbol{Q}$, whose value on each loop depends only on its homotopy class, form a Hopf subalgebra $\pi^{1}$ of $Q$. Evidently $\pi^{1}$ consists of $K$-valued functions on the fundamental group $\pi_{1}(\mathfrak{M})$.

Received by the editors March 15, 1970.

AMS 1970 subject classifications. Primary 53C65; Secondary 57D99.

Key words and phrases. Iterated path integrals, deRham cohomology, Hopf algebras, shuffle algebras, Massey products, nonsolvable fundamental groups.

$\left.{ }^{1}\right)$ Work supported in part by the National Science Foundation under NSF-GP-13145. 
After presenting preliminary material in $\$ \S 1-2$, we construct the Hopf algebra $\pi^{1}$ and investigate its structure. A good number of elements of $\pi^{1}$ can be obtained by using modified Massey products of closed 1-forms. The following results will be given in $\S 3$ :

(i) Let $H(\mathfrak{M})$ be the deRham cohomology of $\mathfrak{M}$, and let $b_{r}=\operatorname{dim}_{K} H^{r}(\mathfrak{M})$. If $b_{1}>1$ and $b_{2}=0$, then $\pi_{1}(\mathfrak{M})$ is not solvable.

(ii) If there exist two closed 1 -forms $w$ and $w^{\prime}$ on $\mathfrak{M}$ with the exterior product $w \wedge w^{\prime}=0$ and if their cohomology classes are linearly independent in $H^{1}(\mathfrak{M})$, then $\pi_{1}(\mathfrak{M})$ is not solvable.

(iii) As a consequence of (ii), if $\mathfrak{M}$ is a compact connected Kähler manifold and if $b^{1,0}>b^{2,0}+1$, where $b^{r, s}=\operatorname{dim} H^{r, s}(\mathfrak{M})$, then $\pi_{1}(\mathfrak{M})$ is not solvable.

(iv) Let $F^{r} \pi^{1}$ denote the subspace of $\pi^{1}$ consisting of those elements which are linear combinations of path integrals that are iterated no more than $r$ times. Then there is an exact sequence

$$
0 \longrightarrow\left(F^{1} \pi^{1}\right)^{2} \cap F^{2} \pi^{1} \stackrel{\subset}{\longrightarrow} F^{2} \pi^{1} \longrightarrow H^{1}(\mathfrak{M}) \wedge_{K} H^{1}(\mathfrak{M}) \stackrel{\lambda}{\longrightarrow} H^{2}(\mathfrak{M})
$$

where $\lambda$ is the cup product.

(v) As a consequence, if $\left(F^{1} \pi^{1}\right)^{2}$ does not contain $F^{2} \pi^{1}$, then $\pi_{1}(\mathfrak{M})$ is not abelian.

In $\S 4$, we determine the algebraic structure of the algebras $\boldsymbol{P}$ and $\boldsymbol{Q}$. As a matter of fact, the algebra $P=P(A)$ and the Hopf algebra $Q=Q(A)$ can be defined for an arbitrary commutative algebra $A$ equipped with a derivation and an augmentation.

In $§ 5$, we relate this work to previous works of algebraic nature [7]-[10] by briefly mentioning functorial characterizations of $P$ and $Q$ and the construction of a Hopf algebra $\pi^{1}(A)$.

As far as the author knows, iterated path integration on manifolds has been systematically investigated by only a few. H. H. Johnson has considered iterated path integrals in connection with prolongations [12]. A. N. Parsin's work [16] is a study of iterated path integrals on Riemann surfaces. In the author's earlier works [1]-[5], iterated path integrals are studied mostly from a group and Lie theoretical point of view.

All functions and forms on the manifold $\mathfrak{M}$ will be understood to be $C^{\infty}$.

All vector spaces and algebras will be over the ground field $K$. The word "morphism" will be used instead of "homomorphism". Every algebra is assumed to possess 1 , and every morphism of algebras preserves 1 .

\section{Paths and integrals.}

1.1. We shall not distinguish two paths which differ only by a reparametrization. The product $\alpha \beta$ is defined to be the path $\alpha$ followed by $\beta$, and the inverse $\gamma=\alpha^{-1}$ of $\alpha$ is given by $\gamma(t)=\alpha(1-t)$.

Denote by $G$ the semigroup of loops at the base point $p$. 
1.2. Two paths are said to be elementarily equivalent if one can be written in the form $\alpha \beta \beta^{-1} \gamma$ and the other in the form $\alpha \gamma$. (Either $\alpha$ or $\gamma$ is allowed to be the empty path.) If $\alpha_{1}, \ldots, \alpha_{r}$ is a finite sequence of paths such that $\alpha_{i}$ and $\alpha_{i+1}$ are elementarily equivalent, $i=1, \ldots, r-1$, then we say that $\alpha_{1}$ and $\alpha_{r}$ are equivalent. Denote by $\bar{\alpha}$ the equivalence class of $\alpha$. Define $\bar{\alpha} \bar{\beta}=\overline{\alpha \beta}$. Then

$$
\bar{\alpha}^{-1}=\overline{\alpha^{-1}} \text {. }
$$

Denote by $\bar{G}$ the group of equivalence classes of loops at $p$.

1.3. By a piecewise regular path, we mean a path with nonvanishing tangent vectors. A reduced path is defined to be a piecewise regular path that is not of the type $\alpha \beta \beta^{-1} \gamma$. We shall include the empty path as a reduced path, and any piecewise regular path of the type $\gamma \gamma^{-1}$ will be taken to be equivalent to the empty path.

1.4. The value of an iterated path integral $\int_{\alpha} w_{1} \cdots w_{r}$ does not depend on the parametrization of $\alpha$ and, as a matter of fact, depends only on the equivalence class $\bar{\alpha}$. (See [4, (2.1)-(2.3)].) The following lemma was proved in [4]:

If $\alpha$ is a nonempty reduced (piecewise regular) path, then there exist 1-forms $w_{1}, \ldots, w_{r}$ such that

$$
\int_{\alpha} w_{1} \cdots w_{r} \neq 0
$$

As a consequence, it was also proved that every piecewise regular path is equivalent to one and only one reduced path.

The next theorem provides an answer to the question how well iterated path integrals separate paths.

THEOREM. Two piecewise regular paths $\alpha$ and $\beta$ are equivalent if and only if $\int_{\alpha} w_{1} \cdots w_{r}=\int_{\beta} w_{1} \cdots w_{r}$ for any 1 -forms $w_{1}, \ldots, w_{r}, r \geqq 1$.

Proof. It remains to show the sufficiency part of the theorem. We may assume that $\int_{\alpha} w_{1} \cdots w_{r} \neq 0$ for some $w_{1}, \ldots, w_{r}$. Otherwise both $\alpha$ and $\beta$ are equivalent to the empty reduced path, and the theorem holds. Given a function $f$ on $\mathfrak{M}$, we obtain from (1.5.2)

$$
\int_{\alpha}\left(f w_{1}\right) w_{2} \cdots w_{r}=f(\alpha(0)) \int_{\alpha} w_{1} \cdots w_{r}+\int_{\alpha} d f w_{1} \cdots w_{r} .
$$

An analogous formula also holds for $\beta$. It follows that $f(\alpha(0))=f(\beta(0))$, which means $\alpha(0)=\beta(0)$.

The path $\beta^{-1} \alpha$ is now piecewise regular. Using (1.6.1) and (1.6.2), verify that every iterated path integral vanishes along $\beta^{-1} \alpha$, which implies that $\beta^{-1} \alpha$ is equivalent to the empty path. Hence $\alpha$ and $\beta$ are equivalent.

1.5. Let $\alpha$ be a path initiating from $p$. Iterated path integrals possess the following formal properties:

$$
\int_{\alpha} w_{1} \cdots w_{r} \int_{\alpha} w_{r+1} \cdots w_{r+s}=\sum_{\sigma} \int_{\alpha} w_{\sigma 1} \cdots w_{\sigma(r+s)}
$$


summing over all $(r, s)$-shuffles, i.e. permutations $\sigma$ of $r+s$ letters with $\sigma^{-1} 1<\cdots<\sigma^{-1} r$ and $\sigma^{-1}(r+1)<\cdots<\sigma^{-1}(r+s)$.

If $f$ is a function, then

$$
\begin{aligned}
\int_{\alpha} w_{1} \cdots w_{r}(f w) w_{r+1} \cdots w_{r+s}= & f(p) \int_{\alpha} w_{1} \cdots w_{r} w w_{r+1} \cdots w_{r+s} \\
& +\int_{\alpha}\left(\int_{\alpha^{t}} w_{1} \cdots w_{r} \int_{\alpha^{t}} d f\right) w w_{r+1} \cdots w_{r+s}
\end{aligned}
$$

The formula (1.5.2) follows from the fact that $f(\alpha(t))=f(p)+\int_{\alpha^{t}} d f$. The formula (1.5.1) was observed by Ree [17]. Its verification can be illustrated by the following particular cases:

$$
\begin{aligned}
\int_{\alpha} w_{1} \int_{\alpha} w_{2} & =\int_{0}^{1}\left[\left(\int_{\alpha^{t}} w_{1}\right) w_{2}(\alpha(t), \dot{\alpha}(t))+\left(\int_{\alpha^{t}} w_{2}\right) w_{1}(\alpha(t), \dot{\alpha}(t))\right] d t \\
& =\int_{\alpha}\left(w_{1} w_{2}+w_{2} w_{1}\right) \\
\int_{\alpha} w_{1} \int_{\alpha} w_{2} w_{3} & =\int_{0}^{1}\left[\left(\int_{\alpha^{t}} w_{1} \int_{\alpha^{t}} w_{2}\right) w_{3}(\alpha(t), \dot{\alpha}(t))+\left(\int_{\alpha^{t}} w_{2} w_{3}\right) w_{1}(\alpha(t), \dot{\alpha}(t))\right] d t \\
& =\int_{\alpha}\left(w_{1} w_{2} w_{3}+w_{2} w_{1} w_{3}+w_{2} w_{3} w_{1}\right) ; \quad \\
\int_{\alpha} w_{1} w_{2} \int_{\alpha} w_{3} w_{4} & =\cdots=\int_{\alpha}\left(w_{1} w_{2} w_{3} w_{4}+w_{1} w_{3} w_{2} w_{4}+w_{1} w_{3} w_{4} w_{2}+w_{3} w_{1} w_{2} w_{4}\right.
\end{aligned}
$$

1.6. The next two formulas relate iterated path integrals to the multiplication and the inverse of paths.

$$
\begin{aligned}
\int_{\alpha \beta} w_{1} \cdots w_{r}= & \int_{\alpha} w_{1} \cdots w_{r}+\cdots+\int_{\alpha} w_{1} \cdots w_{i} \int_{\beta} w_{i+1} \cdots w_{r}+\int_{\beta} w_{1} \cdots w_{r} \\
& \int_{\alpha^{-1}} w_{1} \cdots w_{r}=(-1)^{r} \int_{\alpha} w_{r} \cdots w_{1} .
\end{aligned}
$$

A verification of (1.6.1) was given in [3]. For (1.6.2), set $\gamma=\alpha^{-1}$ and $\alpha_{t}=\alpha \mid[1-t, 1]$. Then $\gamma^{t}=\left(\alpha_{t}\right)^{-1}$. It suffices to show that by induction on $r$

$$
(-1)^{r}(d / d t) \int_{\gamma^{t}} w_{1} \cdots w_{r}=(d / d t) \int_{\alpha_{t}} w_{r} \cdots w_{1} .
$$

In fact, the left-hand side is equal to

$$
\begin{aligned}
-(-1)^{r}\left(\int_{\gamma^{t}} w_{1} \cdots w_{r-1}\right) w_{r}(\alpha(1-t), & \dot{\alpha}(1-t)) \\
= & \left(\int_{\alpha_{t}} w_{r-1} \cdots w_{1}\right) w_{r}(\alpha(1-t), \dot{\alpha}(1-t)),
\end{aligned}
$$


while the right-hand side is equal to

$$
\begin{array}{r}
(d / d t) \int_{1-t}^{1} \int_{1-t}^{s_{1}} \cdots \int_{1-t}^{s_{r-1}} w_{r}\left(\alpha\left(s_{r}\right), \dot{\alpha}\left(s_{r}\right)\right) \cdots w_{1}\left(\alpha\left(s_{1}\right), \dot{\alpha}\left(s_{1}\right)\right) d s_{r} \cdots d s_{1} \\
=\left(\int_{\alpha_{t}} w_{r-1} \cdots w_{1}\right) w_{r}(\alpha(1-t), \dot{\alpha}(1-t)) .
\end{array}
$$

2. The filtration of algebras of path integrals.

2.1. Denote by $T(M)=T^{0}(M)+T^{1}(M)+\cdots$ the tensor algebra of $M$ over $K$, where $T^{r}(M)$ is the $r$-fold tensor product of $M$ which is the vector space of $C^{\infty}$ 1-forms. For simplicity, we shall write

$$
w_{1} \cdots w_{r}=w_{1} \otimes \cdots \otimes w_{r} \in T^{r}(M), \quad r \geqq 1 .
$$

Set $w_{1} \cdots w_{r}=1$ when $r=0$.

If the tensor multiplication of $T(M)$ is replaced by the shuffle multiplication 。 which is given by

$$
w_{1} \cdots w_{r} \circ w_{r+1} \cdots w_{r+s}=\sum_{\sigma} w_{\sigma 1} \cdots w_{\sigma(r+s)}
$$

summing over all $(r, s)$-shuffles $\sigma$, we obtain from $T(M)$ a commutative algebra Sh $(M)$, which will be called the shuffle algebra of $M$.

2.2. There is a surjective linear map

$$
\int_{p}: \operatorname{Sh}(M) \rightarrow P
$$

given by $1 \mapsto 1$ and, for $r \geqq 1$,

$$
w_{1} \cdots w_{r} \mapsto \int_{p} w_{1} \cdots w_{r}
$$

It follows from (1.5.1) that the linear map $\int_{p}$ is a morphism of algebras.

Denote by $\varepsilon_{A}: A \rightarrow K$ the evaluation map at $p$ so that $\varepsilon_{A} f=f(p)$. The formula (1.5.2) implies that the kernel of $\int_{p}$ contains the ideal $I$ generated by all elements of the type

$$
\begin{array}{r}
w_{1} \cdots w_{r}(f w) w_{r+1} \cdots w_{r+s}-\left(\varepsilon_{A} f\right) w_{1} \cdots w_{r} w w_{r+1} \cdots w_{r+s} \\
-\left(w_{1} \cdots w_{r} \circ d f\right) w w_{r+1} \cdots w_{r+s}
\end{array}
$$

for $r \geqq 0, s \geqq 0$. (Such elements not only generate the ideal $I$ but also span $I$ as a vector space. See [7].)

Define $P$ to be the quotient algebra $\operatorname{Sh}(M) / I$. Then there is a canonical surjection $\boldsymbol{P} \rightarrow \boldsymbol{P}$.

2.3. Similarly there is a surjective morphism of algebras

$$
\oint_{p}: \operatorname{Sh}(M) \rightarrow Q
$$


Since $\oint_{p} d f=0$, the kernel of $\oint_{p}$ will not only contain the ideal $I$ but also $d A \subset T^{1}(M) \subset \operatorname{Sh}(M)$. Denote by $(d A)$ the ideal generated by $d A$ in Sh $(M)$. Let $Q$ be the quotient algebra $\mathrm{Sh}(M) /(I+(d A))$. Then $Q$ can be also taken as a quotient algebra of $P$, and there is a canonical morphism of algebras $Q \rightarrow \boldsymbol{Q}$.

The construction of $P$ and $Q$ is described in detail in [7], where they are respectively denoted by $\operatorname{Sh}(d, p)$ and $\operatorname{Shc}(d, p)$.

We shall keep on using $\circ$ to denote the respective multiplications in $P$ and $Q$.

2.4. The shuffle algebra $\operatorname{Sh}(M)$ has an ascending filtration by $F^{r} \mathrm{Sh}(M)$ $=T^{0}(M)+\cdots+T^{r}(M), r \geqq 0$. Denote by $F^{r} P\left(\right.$ resp. $\left.F^{r} P, F^{r} Q, F^{r} Q\right)$ the image of $F^{r}$ Sh $(M)$ under the canonical morphism. Thus the algebras $P, P, Q$ and $Q$ are filtered. Observe that $\boldsymbol{F}^{\boldsymbol{P}} \boldsymbol{P}$ or $\boldsymbol{F}^{r} \boldsymbol{Q}$ consists of linear combinations of path integrals which are iterated not more than $r$ times.

2.5. Given a path $\alpha$, the path integral $\int_{\alpha} u$ is now defined for every element $u$ of Sh $(M)$ with $\int_{\alpha} 1=1$. Write $\langle u, \alpha\rangle=\int_{\alpha} u$.

Let $\varepsilon=\varepsilon_{\mathrm{Sh}(M)}: \operatorname{Sh}(M) \rightarrow K$ be the morphism of algebras given by $w_{1} \cdots w_{r} \mapsto 0$, $r \geqq 1$, i.e. $\varepsilon u=\langle u, e\rangle$ where $e$ is the constant path at $p$.

Definition. A path $\alpha$ is said to be at least of order $r$ if $\langle u, \alpha\rangle=\varepsilon u$, $\forall u \in F^{r-1} \operatorname{Sh}(M), r \geqq 1$. Every path is at least of order 1 . If a path is at least of order 2, then it is a loop.

Denote by $F_{r} G$ the set of loops at $p$ which are at least of order $r$. Let $F_{r} \bar{G}$ be the set of equivalence classes $\bar{\alpha}, \forall \alpha \in F_{r} G$.

Observe that a path $\alpha$ is at least of order $r$ if and only if $\langle u, \alpha\rangle=0$, $\forall u \in F^{r-1} \operatorname{Sh}(M) \cap \operatorname{Ker} \varepsilon$.

Lemma. Let $\alpha \in F_{r} G$, and let $\beta$ be a path from $p$ at least of order $s$. If $u \in F^{r+s-1} \operatorname{Sh}(M) \cap \operatorname{Ker} \varepsilon$, then

$$
\langle u, \alpha \beta\rangle=\langle u, \alpha\rangle+\langle u, \beta\rangle .
$$

Proof. If $u=w_{1} \cdots w_{l}, l<r+s$, it follows from (1.6.1) that $\langle u, \alpha \beta\rangle=\langle u, \alpha\rangle$ $+\langle u, \beta\rangle$. Q.E.D.

Corollary. If $\alpha \in F_{r} G$ and $u \in F^{2 r-1} \operatorname{Sh}(M) \cap \operatorname{Ker} \varepsilon$, then

$$
\left\langle u, \alpha^{-1}\right\rangle=-\langle u, \alpha\rangle .
$$

It follows from the above lemma that $F_{r} \bar{G}$ (resp. $F_{r} G$ ) is a subgroup (resp. subsemigroup) of $\bar{G}$ (resp. $G$ ).

2.6. For $\alpha, \beta \in G$, define $[\alpha, \beta]=\alpha \beta \alpha^{-1} \beta^{-1}$.

Lemma. If $\alpha \in F_{r} G$ and $\beta \in F_{s} G$, then $[\alpha, \beta] \in F_{r+s} G$ and

$$
\begin{aligned}
& \left\langle w_{1} \cdots w_{r+s},[\alpha, \beta]\right\rangle \\
& \quad=\left\langle w_{1} \cdots w_{r}, \alpha\right\rangle\left\langle w_{r+1} \cdots w_{r+s}, \beta\right\rangle-\left\langle w_{1} \cdots w_{s}, \beta\right\rangle\left\langle w_{s+1} \cdots w_{r+s}, \alpha\right\rangle .
\end{aligned}
$$


Proof. Set $l=r+s$. Observe first that

(a) $\sum_{0 \leqq i \leqq r}\left\langle w_{1} \cdots w_{i}, \alpha\right\rangle\left\langle w_{i+1} \cdots w_{l}, \alpha^{-1}\right\rangle=0$;

(b) $\left\langle w_{1} \cdots w_{i}, \alpha\right\rangle\left\langle w_{i+1} \cdots w_{l}, \beta^{-1}\right\rangle=0$ when $i \neq r$;

(c) $\left\langle w_{1} \cdots w_{i}, \beta\right\rangle\left\langle w_{i+1} \cdots w_{l}, \alpha^{-1}\right\rangle=0$ when $i \neq s$.

Using the above formulas as well as 2.5 , we have

$$
\begin{aligned}
\left\langle w_{1} \cdots w_{l},[\alpha, \beta]\right\rangle= & \sum_{0 \leqq i \leqq l}\left\langle w_{1} \cdots w_{i}, \alpha \beta\right\rangle\left\langle w_{l+1} \cdots w_{l}, \alpha^{-1} \beta^{-1}\right\rangle \\
= & \left\langle w_{1} \cdots w_{l}, \alpha\right\rangle+\left\langle w_{1} \cdots w_{r}, \alpha\right\rangle\left\langle w_{r+1} \cdots w_{l}, \beta\right\rangle+\left\langle w_{1} \cdots w_{l}, \beta\right\rangle \\
& +\cdots+\left(\left\langle w_{1} \cdots w_{l}, \alpha\right\rangle+\left\langle w_{1} \cdots w_{i}, \beta\right\rangle\right) \\
& \quad \times\left(\left\langle w_{i+1} \cdots w_{l}, \alpha^{-1}\right\rangle+\left\langle w_{i+1} \cdots w_{l}, \beta^{-1}\right\rangle\right) \\
& +\cdots+\left\langle w_{1} \cdots w_{l}, \alpha^{-1}\right\rangle+\left\langle w_{1} \cdots w_{r}, \alpha^{-1}\right\rangle\left\langle w_{r+1} \cdots w_{l}, \beta^{-1}\right\rangle \\
& +\left\langle w_{1} \cdots w_{l}, \beta^{-1}\right\rangle \\
= & \left\langle w_{1} \cdots w_{r}, \alpha\right\rangle\left\langle w_{r+1} \cdots w_{l}, \beta\right\rangle-\left\langle w_{1} \cdots w_{s}, \beta\right\rangle\left\langle w_{s+1} \cdots w_{l}, \alpha\right\rangle .
\end{aligned}
$$

If $l\left\langle r+s\right.$, the same computation as above will lead to $\left\langle w_{1} \cdots w_{l},[\alpha, \beta]\right\rangle=0$.

Proposition. $F_{r} \bar{G}$ is a normal subgroup of $\bar{G}$, and

$$
\left[F_{r} \bar{G}, F_{s} \bar{G}\right] \subset F_{r+s} \bar{G} .
$$

Proof. It remains to show that, if $\alpha \in F_{r} G$ and $\beta \in G$, then $\beta \alpha \beta^{-1} \in F_{r} G$. In fact, for $u \in F^{r-1} \operatorname{Sh}(M) \cap \operatorname{Ker} \varepsilon$, we have

$$
\left\langle u, \beta \alpha \beta^{-1}\right\rangle=\langle u,[\beta, \alpha] \alpha\rangle=\langle u,[\beta, \alpha]\rangle+\langle u, \alpha\rangle=0 .
$$

REMARK. The group $\bar{G}$ has now a descending filtration by normal subgroups

$$
\bar{G}=F_{1} \bar{G} \supset F_{2} \bar{G} \supset \cdots \text {. }
$$

\section{Hopf algebra $\pi^{1}$.}

3.1. Definition. An element $\int_{p} u \in \boldsymbol{P}$ is said to be independent of paths, if, for every path $\alpha$ from $p, \int_{\alpha} u$ depends only on the homotopy class of the path. The totality of such elements forms a subalgebra $\Gamma$ of $\boldsymbol{P}$. Similarly define elements $\oint_{p} u \in Q$ that are independent of loops, and denote by $\pi^{1}=\pi^{1}(\mathfrak{M}, p)$ the subalgebra of $\boldsymbol{Q}$ consisting of all such elements.

There is a canonical morphism of algebras $\Gamma \rightarrow \pi^{1}$ given by $\int_{p} u \mapsto \oint_{p} u$ and containing all $\int_{p} d f$ in the kernel.

If $w$ is a closed 1-form, obviously $\int_{p} w$ is independent of paths. The next procedure gives us further elements of $\Gamma$.

Let $w_{1}, \ldots, w_{r}, r \geqq 2$, be closed 1 -forms. By an extended defining system for a Massey product of $w_{1}, \ldots, w_{r}$, we mean a family of 1 -forms $w_{12}, w_{23}, \ldots$, $w_{r-1} ; w_{123}, w_{234}, \ldots, w_{r-2 r-1 r} ; \ldots ; w_{12 \ldots r}$ such that

$$
\begin{aligned}
& w_{1} \wedge w_{2}+d w_{12}=0, \ldots, \quad w_{r-1} \wedge w_{r}+d w_{r-1 r}=0 \\
& w_{1} \wedge w_{23}+w_{12} \wedge w_{3}+d w_{123}=0, \ldots ; \ldots ; \\
& w_{1} \wedge w_{2 \ldots r}+w_{12} \wedge w_{3 \ldots r}+\cdots+w_{1 \ldots r-1} \wedge w_{r}+d w_{1 \ldots r}=0 .
\end{aligned}
$$

(See [13], [14].) 
We define the following elements of Sh $(M): u_{1}=w_{1}, u_{12}=u_{1} w_{1}+w_{12}, \ldots, u_{1} \ldots r$ $=u_{1 \ldots r-1} w_{r}+u_{1 \ldots r-2} w_{r-1}+\cdots+w_{1 \ldots r}$.

Note that $\int_{p} u_{1 \ldots r} \equiv \int_{p} w_{1} \cdots w_{r} \bmod F^{r-1} P$.

THEOREM. $\int_{p} u_{1 \ldots r}$ is independent of paths.

Proof. We use induction on $r$. The case of $r=1$ is clear. For $1 \leqq s<r$, the integral $\int_{p} u_{s}$ is independent of paths and lifts to a function $f_{s}$ on the universal covering manifold $\tilde{\mathfrak{M}}$ with $\tilde{f}_{s}(\tilde{p})=0$. Moreover

$$
d \tilde{f}_{s}=\tilde{f}_{s-1} \tilde{w}_{s}+\tilde{f}_{s-2} \tilde{w}_{s-1 s}+\cdots+\tilde{w}_{1 \ldots s}
$$

It remains to show that the 1 -form

$$
\tilde{w}=\tilde{f}_{r-1} \tilde{w}_{r}+\tilde{f}_{r-2} \tilde{w}_{r-1}+\cdots+\tilde{w}_{1 \ldots r}
$$

is closed on $\tilde{\mathfrak{M}}$. In fact

$$
\begin{aligned}
d \tilde{w}= & d \tilde{f}_{r-1} \wedge \tilde{w}_{r}+d \tilde{f}_{r-2} \wedge \tilde{w}_{r-1 r}+\cdots+d \tilde{f}_{1} \wedge \tilde{w}_{2 \ldots r} \\
& +\tilde{f}_{r-2} d \tilde{w}_{r-1,}+\cdots+\tilde{f}_{1} d \tilde{w}_{2 \ldots r}+d \tilde{w}_{1 \ldots r} \\
= & d \tilde{f}_{r-1} \wedge \tilde{w}_{r}+\cdots+d \tilde{f}_{1} \wedge \tilde{w}_{2 \ldots r}-\tilde{f}_{r-2} \tilde{w}_{r-1} \wedge \tilde{w}_{r} \\
& -\cdots-\left(\tilde{w}_{1 \ldots r-1} \wedge \tilde{w}_{r}+\cdots+\tilde{w}_{1} \wedge \tilde{w}_{2 \ldots r}\right) \\
= & \left(d \tilde{f}_{r-1}-\tilde{f}_{r-2} \tilde{w}_{r-1}-\cdots-\tilde{w}_{1 \ldots r-1}\right) \wedge \tilde{w}_{r} \\
& +\cdots+\left(d \tilde{f}_{1}-\tilde{w}_{1}\right) \wedge \tilde{w}_{2 \ldots r} \\
= & 0 .
\end{aligned}
$$

CoROllary. If $w_{1}, \ldots, w_{r}$ are closed 1-forms such that $w_{1} \wedge w_{2}=w_{2} \wedge w_{3}=\cdots$ $=w_{r-1} \wedge w_{r}=0$, then $\int_{p} w_{1} \cdots w_{r}$ is independent of paths.

Proof. Set $w_{12}=w_{23}=\cdots=w_{1 \ldots r}=0$.

Corollary. Assume that $H^{2}(\mathfrak{M})=0$. If $w_{1}, \ldots, w_{r}$ are closed 1-forms, whose cohomology classes are such that $\bar{w}_{1} \wedge \bar{w}_{2}=\bar{w}_{2} \wedge \bar{w}_{3}=\cdots=\bar{w}_{r-1} \wedge \bar{w}_{r}=0$, then there exists $\int_{p} u \in \boldsymbol{P}$ such that $\int_{p} u$ is independent of paths and

$$
\int_{p} u=\int_{p} w_{1} \cdots w_{r} \bmod F^{r-1} P
$$

Proof. We only need to solve the equations (3.1.1) stepwise for $w_{12}, \ldots, w_{r-1 r}$; $\ldots ; w_{1} \ldots .$. Since $w_{1} \wedge w_{2}, \ldots, w_{r-1} \wedge w_{r}$ are closed 2-forms, they are also exact. Thus $w_{12}, \ldots, w_{r-1}$, can be found. Observe that

$$
d\left(w_{1} \wedge w_{23}+w_{12} \wedge w_{3}\right)=w_{1} \wedge w_{2} \wedge w_{3}-w_{1} \wedge w_{2} \wedge w_{3}=0 .
$$

Thus $w_{123}$ exists, and so on. 
3.2. THEOREM. The fundamental group $\pi_{1}(\mathfrak{M})$ is not solvable if one of the following two conditions holds:

(a) $H^{2}(\mathfrak{M})=0$ and $b_{1}=\operatorname{dim}_{K} H^{1}(\mathfrak{M})>1$.

(b) There exist two closed 1-forms $w$ and $w^{\prime}$ on $\mathfrak{M}$ such that $w \wedge w^{\prime}=0$ and $\bar{w}$ and $\bar{w}^{\prime}$ are linearly independent in $H^{1}(\mathfrak{M})$.

Proof. In the case (a), choose two closed 1-forms $w$ and $w^{\prime}$ such that $\bar{w}$ and $\bar{w}^{\prime}$ are linearly independent in $H^{1}(\mathfrak{M})$. In both cases, we may assume that there exist $\alpha, \beta \in G$ such that

$$
\langle w, \alpha\rangle=\left\langle w^{\prime}, \beta\right\rangle=1, \quad\langle w, \beta\rangle=\left\langle w^{\prime}, \alpha\right\rangle=0 .
$$

Write $\left[\beta \alpha^{1}\right]=[\beta, \alpha], \ldots,\left[\beta \alpha^{r}\right]=\left[\left[\beta \alpha^{r-1}\right], \alpha\right], \ldots$ Set

$$
\begin{aligned}
& \gamma_{1}=\left[\beta \alpha^{1}\right], \quad \gamma_{2}=\left[\gamma_{1},\left[\beta \alpha^{2}\right]\right], \\
& \gamma_{3}=\left[\gamma_{2},\left[\left[\beta \alpha^{3}\right],\left[\beta \alpha^{4}\right]\right]\right], \\
& \gamma_{4}=\left[\gamma_{3},\left[\left[\left[\beta \alpha^{5}\right],\left[\beta \alpha^{6}\right]\right],\left[\left[\beta \alpha^{7}\right],\left[\beta \alpha^{8}\right]\right]\right]\right],
\end{aligned}
$$

Our aim is to show that none of these $\gamma_{1}, \gamma_{2}, \ldots$ is null homotopic so that the group $\pi_{1}(\mathfrak{M})$ is not solvable.

Write $w^{r}=w \cdots w$ ( $r$ times) and, for $m<n$,

$$
v_{m n}=w^{\prime} w^{m} w^{\prime} w^{m+1} \cdots w^{\prime} w^{n}
$$

Using 2.6, we verify by induction on $r$ that

$$
\left\langle w^{\prime} w^{r},\left[\beta \alpha^{r}\right]\right\rangle=1 .
$$

Let $v=w_{1} \cdots w_{r+1}$ such that each $w_{i}$ is either $w$ or $w^{\prime}$. If $w^{\prime}$ occur more than once, then

$$
\left\langle v,\left[\beta \alpha^{r}\right]\right\rangle=0 .
$$

Use the above comments and 2.6 to verify that

$$
\begin{aligned}
& \left\langle v_{12}, \gamma_{2}\right\rangle=\left\langle w^{\prime} w,\left[\beta \alpha^{1}\right]\right\rangle\left\langle w^{\prime} w^{2},\left[\beta \alpha^{2}\right]\right\rangle=1, \\
& \left\langle v_{14}, \gamma_{3}\right\rangle=\left\langle v_{12}, \gamma_{2}\right\rangle\left\langle v_{34},\left[\left[\beta \alpha^{3}\right],\left[\beta \alpha^{4}\right]\right]\right\rangle=1,
\end{aligned}
$$

According to the corollaries in 3.1 , there exists $\int_{p} u_{m n} \in \boldsymbol{P}$ which is independent of paths such that

$$
\int_{p} u_{m n} \equiv \int_{p} v_{m n} \bmod F^{l-1} \boldsymbol{P}
$$

where $l=\frac{1}{2}(m+n+2)(n-m+1)$ is the "length" of $v_{m n}$, e.g. $\int_{p} u_{12} \equiv \int_{p} v_{12} \bmod F^{4} P$, $\int_{p} u_{14} \equiv \int_{p} v_{14} \bmod F^{13} P$. Hence

$$
\begin{aligned}
& \left\langle u_{12}, \gamma_{2}\right\rangle=\left\langle v_{12}, \gamma_{2}\right\rangle=1, \\
& \left\langle u_{14}, \gamma_{3}\right\rangle=1, \quad \cdots,
\end{aligned}
$$

and $\gamma_{2}, \gamma_{3}, \ldots$ are not null homotopic. 
We may conclude from this theorem that the fundamental group of any closed Riemann surface of genus $>1$ is not solvable and that the group of any link having two or more components in $R^{3}$ is not solvable.

If $\mathfrak{M}$ is a compact connected Kählerian manifold, let $b^{p, q}=\operatorname{dim} H^{p, q}(\mathfrak{M})$, which is the dimension of the space of holomorphic $(p+q)$-forms of type $(p, q)$. Then the condition (b) of the theorem will be satisfied when $b^{1,0}$ is large compared with $b^{2,0}$. The inequality in the next assertion may not be the best possible but serves as an illustration.

COROLLARY. If $\mathfrak{M}$ is a compact connected Kählerian manifold and if $b^{1,0}>b^{2,0}+1$, then $\pi_{1}(\mathfrak{M})$ is not solvable.

Proof. Let $w_{1}, \ldots, w_{m}$ be linearly independent holomorphic 1-forms, where $m=b^{1,0}$. Then the holomorphic 2-forms $w_{1} \wedge w_{2}, w_{1} \wedge w_{3}, \ldots, w_{1} \wedge w_{m}$ must be linearly dependent, and $w_{1} \wedge w=0$ for some nonzero holomorphic 1 -form.

3.3. The next assertion can be proved in the same manner as Theorem 3.1, in the case of $r=2$.

Proposition. If $w_{i}, w_{i}^{\prime}, i=1, \ldots, r$, are closed 1 -forms and if $w$ is $a$ 1-form of the differentiable manifold $\mathfrak{M}$ such that $\sum w_{i} \wedge w_{i}^{\prime}+d w=0$, then $\int_{p} \sum w_{i} w_{i}^{\prime}+\int_{p} w$ is independent of paths.

Starting from the next section, we are going to investigate the structure of $\pi^{1}$. Note that $\pi^{1}$ is an algebra of $K$-valued functions on $\pi_{1}(\mathfrak{M})$. It is clear that $\operatorname{dim}_{K} \pi^{1} \leqq$ the order of $\pi_{1}(\mathfrak{M})$ and that $\pi^{1}$ is a subspace of the dual space of the group algebra $K \pi_{1}(\mathfrak{M})$.

In order to see that the algebra $\pi^{1}$ arises from some kind of dualization of the fundamental group, we must introduce in $\pi^{1}$ a Hopf algebra structure and view $K \pi_{1}(\mathfrak{M})$ as a Hopf algebra. Then there will be a natural pairing of the two Hopf algebras, and the comultiplication of $\pi^{1}$ dualizes the multiplication of $\pi_{1}(\mathfrak{M})$.

It should be pointed out that there is no loss in replacing a group by its group algebra taken as a Hopf algebra. The group may be retrieved from the group algebra through its Hopf algebra structure.

3.4. Recall that a Hopf algebra $H$ is an algebra equipped with an augmentation (i.e. counit) $\varepsilon_{H}: H \rightarrow K$ and a comultiplication $\zeta_{H}: H \rightarrow H \otimes H$ satisfying the following conditions:

(a) $\zeta_{H}$ is a morphism of algebras.

(b) $\zeta_{H}$ is coassociative, i.e. $\left(\zeta_{H} \otimes 1_{H}\right) \zeta_{H}=\left(1_{H} \otimes \zeta_{H}\right) \zeta_{H}$.

(c) $\zeta_{H}$ is counitary, i.e. $\left(\varepsilon_{H} \otimes 1_{H}\right) \zeta_{H}=\left(1_{H} \otimes \varepsilon_{H}\right) \zeta_{H}=1_{H}$.

The multiplication of $H$ will be denoted by $\mu_{H}: H \otimes H \rightarrow H$, and the canonical morphism of algebras $\eta_{H}: K \rightarrow H$ is known as the unit of $H$. An antipode of a Hopf algebra $H$ is a linear map $j_{H}: H \rightarrow H$ such that

$$
\mu_{H}\left(1_{H} \otimes j_{H}\right) \zeta_{H}=\eta_{H} \varepsilon_{H}=\mu_{H}\left(j_{H} \otimes 1_{H}\right) \zeta_{H} .
$$


If the multiplication (resp. comultiplication) of a Hopf algebra is commutative, then the Hopf algebra is said to be commutative (resp. cocommutative). For a commutative or cocommutative Hopf algebra, the antipode is unique if it exists.

The group algebra $K \bar{G}$ is a cocommutative Hopf algebra, whose comultiplication is given by $\bar{\alpha} \mapsto \bar{\alpha} \otimes \bar{\alpha}, \forall \bar{\alpha} \in \bar{G}$. The antipode of $K \bar{G}$ is given by $\bar{\alpha} \mapsto \bar{\alpha}^{-1}$.

If $M^{\prime}$ is a vector space, then the tensor algebra $T\left(M^{\prime}\right)$ is a cocommutative Hopf algebra, whose comultiplication is the diagonal map $T\left(M^{\prime}\right) \rightarrow T\left(M^{\prime}\right) \otimes T\left(M^{\prime}\right)$ given by $x \mapsto x \otimes 1+1 \otimes x, \forall x \in M^{\prime}$. The antipode of $T\left(M^{\prime}\right)$ is given by

$$
x_{1} \cdots x_{r} \mapsto(-1)^{r} x_{r} \cdots x_{1}, \quad \forall x_{1}, \ldots, x_{r} \in M^{\prime}, \quad r \geqq 1 .
$$

A pairing of two Hopf algebras $H$ and $H^{\prime}$ is a bilinear map $H \times H^{\prime} \rightarrow K$ which sends $\left(h, h^{\prime}\right)$ to an element $\left\langle h, h^{\prime}\right\rangle$ in $K$ such that $\forall h, h_{1}, h_{2} \in H, h^{\prime}, h_{1}^{\prime}, h_{2}^{\prime} \in H^{\prime}$ and $c \in K$,

(3.4.1) $\left\langle h_{1} h_{2}, h^{\prime}\right\rangle=\left\langle h_{1} \otimes h_{2}, \zeta_{H^{\prime}} h^{\prime}\right\rangle$,

(3.4.2) $\left\langle h, h_{1}^{\prime} h_{2}^{\prime}\right\rangle=\left\langle\zeta_{H} h, h_{1}^{\prime} \otimes h_{2}^{\prime}\right\rangle$,

(3.4.3) $\left\langle\eta_{H} c, h^{\prime}\right\rangle=c \varepsilon_{H^{\prime}} h^{\prime}$,

(3.4.4) $\left\langle h, \eta_{H^{\prime}} c\right\rangle=c \varepsilon_{H} h$.

Since Hopf algebras considered in this paper will be equipped with antipodes, we further demand that

$$
\left\langle j_{H} h, h^{\prime}\right\rangle=\left\langle h, j_{H^{\prime}} h^{\prime}\right\rangle .
$$

3.5. A pairing $H \times H^{\prime} \rightarrow K$ is said to be left nondegenerate if $\left\langle h, h^{\prime}\right\rangle=0, \forall h^{\prime} \in H^{\prime}$, implies $h=0$. In this case, the induced pairing $H \otimes H \times H^{\prime} \otimes H^{\prime} \rightarrow K$ is also left nondegenerate. We mention the next assertion.

LEMMA. Let $H^{\prime}$ be a Hopf algebra, and $H$, an algebra equipped with linear maps $\varepsilon_{H}: H \rightarrow K, \zeta_{H}: H \rightarrow H \otimes H$ and $j_{H}: H \rightarrow H$. If there exists a left nondegenerate pairing $H \times H^{\prime} \rightarrow K$ such that (3.4.1)-(3.4.5) hold, then $H$ is a Hopf algebra, and $j_{H}$ is an antipode of $H$.

Motivated by (1.5.3), we equip the shuffle algebra Sh $(M)$ with the linear maps $\zeta_{\mathrm{Sh}(M)}: \operatorname{Sh}(M) \rightarrow \operatorname{Sh}(M) \otimes \operatorname{Sh}(M)$ and $j_{\mathrm{Sh}(M)}: \operatorname{Sh}(M) \rightarrow \operatorname{Sh}(M)$ respectively given by

$$
w_{1} \cdots w_{r} \mapsto \sum_{0 \leqq i \leqq r} w_{1} \cdots w_{i} \otimes w_{i+1} \cdots w_{r}
$$

and $w_{1} \cdots w_{r} \mapsto(-1)^{r} w_{r} \cdots w_{1}, \forall w_{1}, \ldots, w_{r} \in M$.

Choose a basis of $M$ and construct a vector space $M^{\prime}$ and a left nondegenerate pairing $M \times M^{\prime} \rightarrow K$ such that $M^{\prime}$ has a basis dual to that of $M$. The induced pairing

$$
\operatorname{Sh}(M) \times T\left(M^{\prime}\right) \rightarrow K
$$

is given by $\left\langle w_{1} \cdots w_{r}, x_{1} \cdots x_{s}\right\rangle=0$ when $r \neq s$ and

$$
\left\langle w_{1} \cdots w_{r}, x_{1} \cdots x_{r}\right\rangle=\left\langle w_{1}, x_{1}\right\rangle \cdots\left\langle w_{r}, x_{r}\right\rangle
$$


and is left nondegenerate. Verify that (3.4.1)-(3.4.5) hold. Therefore $\mathrm{Sh}(M)$ is a Hopf algebra having an antipode.

3.6. There is a pairing $\operatorname{Sh}(M) \times K \bar{G} \rightarrow K$ given by $\left\langle w_{1} \cdots w_{r}, \bar{\alpha}\right\rangle=\int_{\alpha} w_{1} \cdots w_{r}$. The formulas (3.4.1), (3.4.2) and (3.4.5) follow respectively from (1.5.1), (1.6.1) and (1.6.2), while (3.4.3) and (3.4.4) hold trivially for this pairing. Therefore this is a pairing of Hopf algebras.

There is a left nondegenerate pairing

$$
\boldsymbol{Q} \times K \bar{G} \rightarrow K
$$

given by $\left\langle\oint_{p} u, \bar{\alpha}\right\rangle=\langle u, \alpha\rangle, \forall u \in \operatorname{Sh}(M)$. Thus the induced pairing

$$
\boldsymbol{Q} \otimes \boldsymbol{Q} \times K \bar{G} \otimes K \bar{G} \rightarrow K
$$

is also left nondegenerate.

Define the augmentation $\varepsilon_{Q}: Q \rightarrow K$ by

$$
\varepsilon_{Q} \oint_{p} u=\varepsilon_{\mathrm{Sh}(M)} u,
$$

which is equal to $\oint_{p} u$ evaluated along the constant loop $e$.

Define $\zeta_{\boldsymbol{Q}}: \boldsymbol{Q} \rightarrow \boldsymbol{Q} \otimes \boldsymbol{Q}$ by

$$
\zeta_{Q} \oint_{p} w_{1} \cdots w_{r}=\sum_{0 \leqq i \leqq r} \oint_{p} w_{1} \cdots w_{i} \otimes \oint_{p} w_{i+1} \cdots w_{r} .
$$

Then, $\forall \bar{\alpha}, \bar{\beta} \in \bar{G}$,

$$
\left\langle\zeta_{Q} \oint_{p} u, \bar{\alpha} \otimes \bar{\beta}\right\rangle=\left\langle\oint_{p} u, \bar{\alpha} \bar{\beta}\right\rangle \text {. }
$$

The left nondegeneracy of the pairing (3.6.2) makes $\zeta_{Q}$ a well-defined linear map. A similar argument makes a well-defined linear map out of $j_{\boldsymbol{Q}}: \boldsymbol{Q} \rightarrow \boldsymbol{Q}$ given by

$$
\oint_{p} w_{1} \cdots w_{r} \mapsto(-1)^{r} \oint_{p} w_{r} \cdots w_{1} .
$$

Now verify that (3.4.1)-(3.4.5) hold for the pairing (3.6.1). Hence we reach the next conclusion.

PROPOSITION. With the augmentation $\varepsilon_{Q}$, the comultiplication $\zeta_{Q}$ and the antipode $j_{Q}, \boldsymbol{Q}$ is a Hopf algebra.

Remark. The filtration $F$ of $\boldsymbol{Q}$ is consistent with the multiplication and the comultiplication of $\boldsymbol{Q}$, and $\boldsymbol{Q}$ is therefore a filtered Hopf algebra.

3.7. Lemma. Let $\rho: H^{\prime} \rightarrow H_{1}^{\prime}$ be a morphism of Hopf algebras. If $H \times H^{\prime} \rightarrow K$ is a left nondegenerate pairing of Hopf algebras, then $H_{1}=(\operatorname{Ker} \rho)^{\perp}$ is a Hopf subalgebra of $H$. 
Proof. Write $N=\operatorname{Ker} \rho$. Observe that $H_{1} \otimes H_{1}$ is precisely $\left(N \otimes H^{\prime}+H^{\prime} \otimes N\right)^{\perp}$ with respect to the left nondegenerate pairing $H \otimes H \times H^{\prime} \otimes H^{\prime} \rightarrow K$. We use (3.4.1) and (3.4.2) and the left nondegeneracy of the involved pairings to show that $H_{1}$ is closed under the multiplication and the comultiplication of $H$. Q.E.D.

3.8. Denote by $G_{0}$ the subsemigroup of $G$ of null homotopic loops at $p$. Denote by $\bar{G}_{0}$ the normal subgroup of $\bar{G}$ consisting of all $\bar{\alpha}, \alpha \in G_{0}$. Then $\bar{G} / \bar{G}_{0}$ is the fundamental group $\pi_{1}(\mathfrak{M})=\pi_{1}(\mathfrak{M}, p)$. For $\alpha \in G$, the corresponding element of $\pi_{1}(\mathfrak{M})$ will be denoted by $\hat{\alpha}$.

Definition. Denote by $\pi^{1}=\pi^{1}(\mathfrak{M}, p)$ the subalgebra of $Q$ consisting of all $\oint_{p} u$ such that the value of $\oint_{p} u$ along each loop $\alpha$ at $p$ depends only on $\hat{\alpha} \in \pi_{1}(\mathfrak{M})$.

Observe that $\pi^{1}$ is the subalgebra of $Q$ consisting of elements that vanish on $G_{0}$. It is clear that $j Q^{\pi^{1} \subset \pi^{1}}$.

THEOREM. $\pi^{1}$ is a Hopf subalgebra of $\boldsymbol{Q}$ with antipode.

Proof. Let $N$ denote the kernel of the morphism of Hopf algebras $\rho: K \bar{G} \rightarrow K \pi_{1}(\mathfrak{M})$. Then $N$ is spanned by elements of the type $\bar{\alpha}-\bar{\beta}$ such that the loops $\alpha$ and $\beta$ are homotopic. We have $\pi^{1}=N^{\perp}$ with respect to the pairing (3.6.1). It follows from Lemma 3.7 that $\pi^{1}$ is a Hopf algebra. Q.E.D.

3.9. We are going to treat the question of change of base point. Let $\gamma$ be a path from the base point $p$ to a new base point $q$. Define a morphism of Hopf algebras

$$
\gamma^{*}: Q(\mathfrak{M}, p) \rightarrow Q(\mathfrak{M}, q)
$$

by the formula $\left\langle\gamma^{*} \oint_{p} u, \beta\right\rangle=\left\langle\oint_{p} u, \gamma \beta \gamma^{-1}\right\rangle$ for any loop $\beta$ at $q$. The fact that $\gamma^{*} \oint_{p} u$ belongs to $Q(\mathfrak{M}, q)$ follows from the next formula, which is a consequence of (1.6.1).

$$
\int_{\gamma \beta \gamma^{-1}} w_{1} \cdots w_{r}=\sum_{0 \leqq i \leqq j \leqq r} \int_{y} w_{1} \cdots w_{i} \int_{\beta} w_{i+1} \cdots w_{j} \int_{\gamma^{-1}} w_{j+1} \cdots w_{r} .
$$

Since $\left(\gamma^{-1}\right)^{*}=\gamma^{*-1}, \gamma^{*}$ is an isomorphism. It can be verified that $\gamma^{*}$ preserves the antipode and sends $\pi^{1}(\mathfrak{M}, p)$ bijectively onto $\pi^{1}(\mathfrak{M}, q)$. Let

$$
\gamma^{* *}: \pi^{1}(\mathfrak{M}, p) \approx \pi^{1}(\mathfrak{M}, q)
$$

be the restriction of $\gamma^{*}$.

3.10. We may take both $Q$ and $\pi^{1}$ as covariant functors from the category of pointed $C^{\infty}$ manifolds to that of commutative Hopf algebras. The next assertion can be verified in a routine fashion.

Proposition. If $\phi_{i}: \mathfrak{M}^{\prime} \rightarrow \mathfrak{M}, i=0,1$, are two homotopic $C^{\infty}$ maps, then

$$
\pi^{1}\left(\phi_{0}\right)=\pi^{1}\left(\phi_{1}\right) \gamma^{* *}
$$

where $\gamma$ is a path from $\phi_{0} p^{\prime}$ to $\phi_{1} p^{\prime}, p^{\prime}$ being the base point of $\mathfrak{M}^{\prime}$.

This assertion implies that, if $\mathfrak{M}$ is compact, then $\pi^{1}$, up to an isomorphism, depends only on the homotopy type of $\mathfrak{M}$. It will be interesting to know whether 
$\pi^{1}$ can be actually constructed from $\pi_{1}(\mathfrak{M})$ as a group without reference to the manifold. In other words, is $\pi^{1}$ indeed a contravariant functor from the category of groups to the category of commutative Hopf algebras?

3.11. The Hopf algebra $\pi^{1}$ is filtered by

$$
F^{r} \pi^{1}=F^{r} Q \cap \pi^{1}, \quad r=0,1, \ldots
$$

Clearly $F^{0} \pi^{1}=K$, and $F^{1} \pi^{1}$ consists of all $c+\oint_{p} w$, where $c \in K$ and $w$ is a closed 1 -form. Therefore there is an exact sequence

$$
0 \rightarrow F^{0} \pi^{1} \rightarrow F^{1} \pi^{1} \rightarrow H^{1}(\mathfrak{M}) \rightarrow 0 .
$$

Proposition. An element of $F^{2} Q$ belongs to $\pi^{1}$ if and only if the element can be written in the form

$$
\oint_{p}\left(\sum w_{i} w_{i}^{\prime}+w+c\right)
$$

such that $w_{i}$ and $w_{i}^{\prime}$ are closed 1-forms, and

$$
\sum w_{i} \wedge w_{i}^{\prime}+d w=0
$$

Proof. The sufficiency is implied by Proposition 3.3. The vector space of 1-forms can be written as a direct sum $M_{0} \oplus M_{1}$, where $M_{0}$ is the subspace of exact 1-forms. If $w \in M_{0}$, then

and

$$
\oint_{p} w w^{\prime}=\oint_{p}(d f) w^{\prime}=\oint_{p} f w^{\prime}-f(p) \oint_{p} w^{\prime \prime} \in F^{1} Q
$$

$$
\oint_{p} w^{\prime} w=-\oint_{p}(d f) w^{\prime} \in F^{1} Q
$$

It follows that every element of $F^{2} \pi^{1}$ can be written in the form $\oint_{p} u, u$ $=\sum w_{i} w_{i}^{\prime}+w+c$, such that $w_{i}, w_{i}^{\prime} \in M_{1}$. The coproduct

$$
\zeta_{Q} \oint_{p} u=\oint_{p} u \otimes 1+\sum \oint_{p} w_{i} \otimes \oint_{p} w_{i}^{\prime}+1 \otimes \oint_{p} u
$$

belongs to $\pi^{1} \otimes \pi^{1}$ so that

$$
\sum \oint_{p} w_{i} \otimes \oint_{p} w_{i}^{\prime} \in \pi^{1} \otimes \pi^{1}
$$

This means that the element $\sum w_{i} \otimes w_{i}^{\prime}$ can be written in the form such that $w_{i}$ and $w_{i}^{\prime}$ are closed.

Let $\tilde{\mathfrak{M}}$ be the universal covering space of $\mathfrak{M}$ with a base point $\tilde{p}$ which projects to $p$. Then $\int_{p} w_{i}$ lifts to a function $\tilde{f_{i}}$ on $\tilde{\mathfrak{M}}$, and $w, w_{i}, w_{i}^{\prime}$ lift respectively to 1-forms $\tilde{w}, \tilde{w}_{i}, \tilde{w}_{i}^{\prime}$ on $\tilde{\mathfrak{M}}$. Since $\oint_{p} u \in \pi^{1}$, the 1 -form $\sum \tilde{f}_{i} \tilde{w}_{i}^{\prime}+\tilde{w}$ must be closed so that

$$
d\left(\sum \tilde{f}_{i} w_{i}^{\prime}+\tilde{w}\right)=\sum \tilde{w}_{i} \wedge \tilde{w}_{i}^{\prime}+d \tilde{w}=0 .
$$

Hence $\sum w_{i} \wedge w_{i}^{\prime}+d w=0$. 
3.12. If $w$ is a closed 1 -form, denote by $\bar{w}$ its cohomology class in $H^{1}(\mathfrak{M})$. Denote by $H^{1}(\mathfrak{M}) \wedge_{K} H^{1}(\mathfrak{M})$ the alternating product over $K$. We may regard $H^{1}(\mathfrak{M}) \wedge_{K} H^{1}(\mathfrak{M})$ as a vector space of $K$-valued functions on the Cartesian product $G \times G$ such that, if $\alpha, \beta \in G$ and $\sum \bar{w}_{i} \wedge_{K} \bar{w}_{i}^{\prime} \in H^{1}(\mathfrak{M}) \wedge_{K} H^{1}(\mathfrak{M})$, then

$$
\left(\sum \bar{w}_{i} \wedge_{K} \bar{w}_{i}^{\prime}\right)(\alpha, \beta)=\sum\left\langle w_{i}, \alpha\right\rangle\left\langle w_{i}^{\prime}, \beta\right\rangle-\left\langle w_{i}^{\prime}, \alpha\right\rangle\left\langle w_{i}, \beta\right\rangle
$$

Let $u=\sum w_{i} w_{i}^{\prime}+w+c$ where $w_{i}, w_{i}^{\prime}$ are closed. Define

$$
\nu: F^{2} \pi^{1} \rightarrow H^{1}(\mathfrak{M}) \wedge_{K} H^{1}(\mathfrak{M})
$$

by $\oint_{p} u \rightarrow \sum \bar{w}_{i} \wedge_{K} \bar{w}_{i}^{\prime}$. Then, $\forall \alpha, \beta \in G$, we have

$$
\left(\sum \bar{w}_{i} \wedge_{K} \bar{w}_{i}^{\prime}\right)(\alpha, \beta)=\int_{[\alpha, \beta]} u .
$$

Consequently $\nu$ is well defined.

THEOREM. There is an exact sequence

$$
0 \longrightarrow\left(F^{1} \pi^{1} \circ F^{1} \pi^{1}\right) \cap F^{2} \pi^{1} \stackrel{\subset}{\longrightarrow} F^{2} \pi^{1} \stackrel{\nu}{\longrightarrow} H^{1}(\mathfrak{M}) \wedge_{K} H^{1}(\mathfrak{M}) \stackrel{\lambda}{\longrightarrow} H^{2}(\mathfrak{M})
$$

where $\lambda$ is the cup product.

Proof. The exactness at $H^{1}(\mathfrak{M}) \wedge_{K} H^{1}(\mathfrak{M})$ is an immediate consequence of 3.11. It remains to show the exactness at $F^{2} \pi^{1}$. It is clear that $\left(F^{1} \pi^{1} \circ F^{1} \pi^{1}\right) \cap F^{2} \pi^{1}$ is contained in $\operatorname{Ker} \nu$. We are going to show the inclusion in the other direction.

Let $\oint_{p} u \in \operatorname{Ker} v$ such that $u=\sum w_{i} w_{i}^{\prime}+w+c$. We may assume that $c=0$. According to 3.11, $w_{i}$ and $w_{i}^{\prime}$ can be taken to closed 1-forms, and $w_{i}, w_{i}^{\prime} \in M_{1}$. The fact that $\sum \bar{w}_{i} \wedge_{K} \bar{w}_{i}^{\prime}=0$ implies that $\sum w_{i} w_{i}^{\prime}$ is a linear combination of elements of the type $w^{\prime \prime} w^{\prime}+w^{\prime} w^{\prime \prime}$. Therefore $\oint_{p} \sum w_{i} w_{i}^{\prime}$ is a linear combination of elements of the type $\oint_{p} w^{\prime} \circ \oint_{p} w^{\prime \prime}$, where $w^{\prime}$ and $w^{\prime \prime}$ are closed 1-forms. On the other hand, since $\sum \bar{w}_{i} \wedge \bar{w}_{i}^{\prime}=0, w$ is also a closed 1-form. Hence $\oint_{p} u \in F^{1} \pi^{1} \circ F^{1} \pi^{1}$.

An immediate consequence of this theorem and (3.12.1) is that, if $\pi_{1}(\mathfrak{M})$ is abelian, then $\lambda$ is injective. As pointed out by W. S. Massey, this result is valid for $\mathrm{CW}$ complexes with a finitely generated fundamental group and an arbitrary coefficient field for cohomology. (See a footnote, [11].)

The exactness of the sequence at $F^{2} \pi^{1}$ implies the following assertion, which relates analysis of the manifold with the fundamental group:

Corollary. If $F^{2} \pi^{1}$ is not contained in $F^{1} \pi^{1} \circ F^{1} \pi^{1}$, then $\pi_{1}(\mathfrak{M})$ is not abelian .

4. Identification theorems.

4.1. Recall the algebras $P$ and $Q$ as defined in 2.2 and 2.3. If $u \in \operatorname{Sh}(M)$, denote by $u^{\prime}$ and $u^{\prime \prime}$ respectively the images of $u$ under the canonical surjections $\mathrm{Sh}(M) \rightarrow P$ and $\mathrm{Sh}(M) \rightarrow Q$. 
The MaIN Lemma. If $u \in T^{r}(M), r>1$, is such that $\langle u, \alpha\rangle=0, \forall \alpha \in F_{r} G$, then $u^{\prime} \in F^{r-1} P \circ F^{r-1} P$ and $u^{\prime \prime} \in F^{r-1} Q \circ F^{r-1} Q$.

The proof of this lemma will be given in 4.2. We now prepare for this purpose.

Let $\alpha_{1}, \alpha_{2}, \ldots \in G$. Define, for $r>1$,

$$
\left[\alpha_{1}, \ldots, \alpha_{r}\right]=\left[\left[\alpha_{1}, \ldots, \alpha_{r-1}\right], \alpha_{r}\right] .
$$

For $w_{1}, w_{2}, \ldots \in M$, define $\left[w_{1}\right]=w_{1}$ and, for $r>1$,

$$
\left[w_{1} \cdots w_{r}\right]=\left[w_{1} \cdots w_{r-1}\right] w_{r}-\left[w_{2} \cdots w_{r}\right] w_{1},
$$

which extends to a linear map $T^{r}(M) \rightarrow T^{r}(M)$.

Define the pairing

$$
T^{r}(M) \times K G \otimes \cdots \otimes K G(r \text { times }) \rightarrow K
$$

by $\left\langle\left\langle w_{1} \cdots w_{r}, \alpha_{1} \otimes \cdots \otimes \alpha_{r}\right\rangle\right\rangle=\left\langle w_{1}, \alpha_{1}\right\rangle \cdots\left\langle w_{r}, \alpha_{r}\right\rangle$.

If $\left\langle\left\langle u, \alpha_{1} \otimes \cdots \otimes \alpha_{r}\right\rangle\right\rangle=0, \forall \alpha_{1}, \ldots, \alpha_{r} \in G$, then

$$
u \in \sum_{1 \leqq i \leqq r} T^{i-1}(M) M_{0} T^{r-i}(M)
$$

where $M_{0}$ is the subspace of exact 1-forms of $M$.

Lemma. For $u \in T^{r}(M)$,

$$
\left\langle u,\left[\alpha_{1}, \ldots, \alpha_{r}\right]\right\rangle=\left\langle\left\langle[u], \alpha_{1} \otimes \cdots \otimes \alpha_{r}\right\rangle\right\rangle .
$$

Proof. Use induction on $r \geqq 1$. For $r>1$ and $u=w_{1} \cdots w_{r}$, it follows from 2.6 that

$$
\begin{aligned}
\left\langle u,\left[\alpha_{1}, \ldots, \alpha_{r}\right]\right\rangle= & \left\langle w_{1} \cdots w_{r-1},\left[\alpha_{1}, \ldots, \alpha_{r-1}\right]\right\rangle\left\langle w_{r}, \alpha_{r}\right\rangle \\
& -\left\langle w_{1}, \alpha_{r}\right\rangle\left\langle w_{2} \cdots w_{r},\left[\alpha_{1}, \ldots, \alpha_{r-1}\right]\right\rangle \\
= & \left\langle\left\langle\left[w_{1} \cdots w_{r}\right], \alpha_{1} \otimes \cdots \otimes \alpha_{r}\right\rangle\right\rangle .
\end{aligned}
$$

CoRollaRY. If $u \in T^{r}(M)$ and if $\left\langle u,\left[\alpha_{1}, \ldots, \alpha_{r}\right]\right\rangle=0, \forall \alpha_{1}, \ldots, \alpha_{r} \in G$, then

$$
[u] \in \sum_{0 \leqq i \geqq r} T^{i-1}(M) M_{0} T^{r-i}(M) .
$$

4.2. LemMA. If $u \in T^{i-1}(M) M_{0} T^{r-i}(M), 0<i \leqq r, r \geqq 2$, then $u^{\prime} \in F^{r-1} P$ and $u^{\prime \prime} \in F^{r-1} Q$.

Proof. Set $u_{i}=w_{1} \cdots w_{i-1}(d f) w_{i} \cdots w_{r-1}$ with $\varepsilon_{A} f=f(p)=0$. Verify by induction on $i$ that $u_{i}^{\prime} \in F^{r-1} P, 1 \leqq i \leqq r$, as follows:

$$
\begin{aligned}
& u_{1} \equiv\left(f w_{1}\right) w_{2} \cdots w_{r-1} \bmod I, \\
& u_{i} \equiv w_{1} \cdots\left(f w_{i}\right) \cdots w_{r-1}-u_{1}-\cdots-u_{i-1} \bmod I .
\end{aligned}
$$

LEMMA (R. REE). $r w_{1} \cdots w_{r}=\left[w_{1} \cdots w_{r}\right]+\left[w_{1} \cdots w_{r-1}\right] \circ w_{r}+\cdots+\left[w_{1}\right] \circ w_{2} \cdots w_{r}$.

A proof of the above lemma can be found in [17, p. 212].

Proof of the Main Lemma. According to Ree's lemma,

$$
u \equiv r^{-1}[u] \bmod F^{r-1} \operatorname{Sh}(M) \circ F^{r-1} \operatorname{Sh}(M)
$$


Since $\left[\alpha_{1}, \ldots, \alpha_{r}\right] \in F_{r} G$, it follows from 4.1 that

$$
[u] \in \sum_{1 \leqq i \leqq r} T^{i-1}(M) M_{0} T^{r-i}(M),
$$

which implies $[u]^{\prime} \in F^{r-1} P \subset F^{r-1} P \circ F^{r-1} P$. Hence the lemma is proved.

4.3. Let $V_{r}$ be a subspace of $T^{r}(M)$, and write

$$
V=V_{1}+\cdots+V_{r}+\cdots \subset \operatorname{Sh}(M) .
$$

We demand that the following conditions are satisfied:

(a) The canonical morphism $\operatorname{Sh}(M) \rightarrow P$ restricted to $V$ is injective.

(b) If $V_{r}^{\prime}$ is the image of $V_{r}$ in $P$, then

$$
\begin{aligned}
& F^{1} P=V_{1}^{\prime} \oplus M_{0}^{\prime} \oplus F^{0} P, \\
& F^{r} P=V_{r}^{\prime} \oplus\left(F^{r-1} P \circ F^{r-1} P\right) \cap F^{r} P, \quad r>1,
\end{aligned}
$$

where $M_{0}^{\prime}$ denotes the image of $M_{0} \subset \operatorname{Sh}(M)$ in $P$.

If $S(V)$ denotes the symmetric algebra of $V$, then the inclusion $V \subset \operatorname{Sh}(M)$ gives rise to a morphism of algebras $S(V) \rightarrow \operatorname{Sh}(M)$. Let $\nu$ and $\nu^{\prime \prime}$ be the respective composite morphism of algebras

$$
S(V) \rightarrow \mathrm{Sh}(M) \rightarrow P, \quad S(V) \rightarrow \mathrm{Sh}(M) \rightarrow Q .
$$

Let the morphism of algebras $\nu^{\prime}: S(V) \otimes A \rightarrow P$ be given by

$$
u \otimes f \mapsto(\nu u)\left(\varepsilon_{A} f+\int_{p} d f\right)
$$

We shall regard $V$ as a subspace of $S(V) \otimes A$ through the canonical embedding. 4.4. We make the following observation: Let $x_{1}, \ldots, x_{l}$ be $K$-valued functions on a set $\mathfrak{S}$, and let $x(\mathfrak{S})$ denote the image of a map $\mathfrak{S} \rightarrow K$ given by $s \mapsto\left(x_{1}(s), \ldots, x_{l}(s)\right)$. If $x(\mathfrak{S})$ contains an additive subgroup of $K^{l}$ whose elements span the vector space $K^{l}$, then the functions $x_{1}, \ldots, x_{l}$ are algebraically independent over the field $K$.

LEMmA. If $x_{1}, \ldots, x_{l}$ are linearly independent elements of $V_{r}, r \geqq 1$, then, as functions on $F_{r} G, \nu x_{1}, \ldots, \nu x_{l}$ are algebraically independent over $K$.

Proof. It follows from 2.5 that, for any $\alpha, \beta \in F_{r} G$, we have

$$
\nu x(\alpha \beta)=\nu x(\alpha)+\nu x(\beta)
$$

and $\nu x\left(\alpha^{-1}\right)=-\nu x(\alpha)$. It remains to show that $\nu x\left(F_{r} G\right)$ contains a basis of $K^{l}$. The case of $r=1$ is clear. For $r>1$, we suppose the contrary. Then there exists $u \in V_{r}$, $u \neq 0$, such that $\langle u, \alpha\rangle=0, \forall \alpha \in F_{r} G$. According to the Main Lemma,

$$
u^{\prime} \in\left(F^{r-1} P \circ F^{r-1} P\right) \cap V_{r}^{\prime}
$$

Consequently $u^{\prime}=0$, which implies $u=0$. Q.E.D. 
4.5. Denote by $\chi: A \rightarrow P$ the linear map given by $f \mapsto\left(\varepsilon_{A} f+d f\right)^{\prime}$. Verify that $\chi$ is a morphism of algebras.

THEOREM. The canonical morphism of algebras $\boldsymbol{P} \rightarrow \boldsymbol{P}$ and $Q \rightarrow \boldsymbol{Q}$ are isomorphisms.

Proof. Let $\tilde{\boldsymbol{\nu}}^{\prime}: S(V) \otimes A \rightarrow P$ be the morphism of algebras given by $v \otimes f \mapsto v^{\prime} \circ \chi f, \forall v \in V, f \in A$. Observe that $\nu^{\prime}$ has a factorization

$$
S(V) \otimes A \stackrel{\tilde{\nu}^{\prime}}{\longrightarrow} P \longrightarrow P
$$

We shall establish the isomorphisms

$$
S(V) \otimes A \stackrel{\tilde{\nu}^{\prime}}{\approx} P \approx P
$$

by showing that $\tilde{\nu}^{\prime}$ is surjective and that $\nu^{\prime}$ is injective.

For the surjectiveness of $\tilde{\nu}^{\prime}$, one needs only to show that the image $D_{r}$ of the composite map

$$
S\left(V_{1}+\cdots+V_{r}\right) \otimes A \longrightarrow S(V) \otimes A \stackrel{\tilde{\nu}^{\prime}}{\longrightarrow} P
$$

contains $F^{r} P$. The case of $r=1$ is clear. For $r>1, D_{r}$ is a subalgebra of $P$ and contains both $F^{r-1} P$ and $V_{r}^{\prime}$ and therefore $F^{r} P$.

In order to establish the injectiveness of $\nu^{\prime}$, it suffices to show by induction on $r \geqq 0$ that the composition

$$
\nu_{r}^{\prime}: S\left(V_{1}+\cdots+V_{r}\right) \otimes A \longrightarrow S(V) \otimes A \stackrel{\nu^{\prime}}{\longrightarrow} P
$$

is injective, where $S\left(V_{1}+\cdots+V_{r}\right)=K$ in the case of $r=0$. We first observe that $\nu_{0}^{\prime}$ is precisely the composition

$$
A \stackrel{\chi}{\longrightarrow} P \longrightarrow P
$$

given by $f \mapsto \varepsilon_{A} f+\int_{p} d f$ and is therefore injective.

For $r>0$, there is a canonical isomorphism

$$
S\left(V_{1}+\cdots+V_{r}\right) \otimes A \approx S\left(V_{r}\right) \otimes\left(S\left(V_{1}+\cdots+V_{r-1}\right) \otimes A\right)
$$

so that any $u \in S\left(V_{1}+\cdots+V_{r}\right) \otimes A$ can be written as a polynomial $u=\sum_{m} u_{m} x^{m}$ where $x=\left(x_{1}, \ldots, x_{l}\right)$ are linearly independent elements of $V_{r}, x^{m}=x^{m_{1}} \cdots x_{l}^{m_{l}}$, and each coefficient $u_{m}$ belongs to $S\left(V_{1}+\cdots+V_{r-1}\right) \otimes A$.

According to 2.5 , for any $\alpha \in F_{r} G$ and any path $\beta$ from $p$,

$$
\langle x, \alpha \beta\rangle=\langle x, \alpha\rangle+\langle x, \beta\rangle .
$$

On the other hand, each $u_{m}$ is a linear combination of elements of the type $h(y) \otimes f$, where $f \in A$ and $h(y)$ is a polynomial in $y=\left(y_{1}, \ldots, y_{k}\right)$ such that $y_{1}, \ldots, y_{k}$ are 
linearly independent elements of $V_{1}+\cdots+V_{r-1}$. Observe that, according to 2.5, $\langle y, \alpha \beta\rangle=\langle y, \beta\rangle$ and that the value of the function $\nu_{r}^{\prime}(h(y) \otimes f)$ at $\alpha \beta$ is

$$
h(\langle y, \beta\rangle)\left(\varepsilon_{A} f+\int_{\beta} d f\right)
$$

which coincides with its value on $\beta$. It follows that the value of the function $\nu_{r-1}^{\prime} u_{m}$ at $\alpha \beta$ is identical with its value at $\beta$, which is here written as $\left\langle u_{m}, \beta\right\rangle$. Let $g_{\beta}(x)$ denote the polynomial $\sum_{m}\left\langle u_{m}, \beta\right\rangle x^{m}$. If $\nu_{r}^{\prime} u=0$, then $\nu_{r}^{\prime} u$ evaluated at $\alpha \beta$ is

$$
g_{\beta}(\langle x, \alpha \beta\rangle)=g_{\beta}(\langle x, \alpha\rangle+\langle x, \beta\rangle)=0,
$$

$\forall \alpha \in F_{r} G$. Since $\nu^{\prime} x_{1}, \ldots, \nu^{\prime} x_{l}$, as functions on $F_{r} G$, are algebraically independent over $K$, we have

$$
g_{\beta}(x+\langle x, \beta\rangle)=0
$$

and therefore $g_{\beta}(x)=0$. This means that the functions $v_{r-1}^{\prime} u_{m}$ are identically zero. Since, by the induction hypothesis, $\nu_{r-1}^{\prime}$ is injective, we have $u_{m}=0$ and hence $u=0$.

The above proof is also valid for the isomorphisms $S(V) \approx Q \approx Q$ if we replace $A$ by $K, P$ by $Q$ and paths by loops. Q.E.D.

Corollary. $S(V) \otimes A \approx P$ and $S(V) \approx Q$.

5. Functorial characterizations. Hereafter, $K$ will denote an arbitrary ground field. We shall use $A$ to denote a $K$-algebra equipped with a derivation, which consists of a $K$-algebra $|A|$, a $|A|$-module $\Omega A$ and a derivation $d_{A}:|A| \rightarrow \Omega A$.

Denote by $\eta_{A}: K \rightarrow|A|$ the canonical morphism of $K$-algebras. We say that $A$ is augmented if there is given an augmentation $\varepsilon_{A}:|A| \rightarrow K$.

Unless otherwise specified, the word "algebra" will mean " $K$-algebra equipped with a derivation". A morphism of algebras $\phi: A \rightarrow A^{\prime}$ consists of a morphism of $K$-algebras $|\phi|:|A| \rightarrow\left|A^{\prime}\right|$ and a $K$-module morphism $\Omega \phi: \Omega A \rightarrow \Omega A^{\prime}$ satisfying the conditions $\Omega \phi d_{A}=d_{A^{\prime}}|\phi|$ and $\Omega \phi(a x)=(|\phi| a) \Omega \phi x, \forall a \in|A|, x \in \Omega A$.

5.1. Definition. An algebra $B$ is exact if the sequence

$$
0 \longrightarrow K \stackrel{\eta_{B}}{\longrightarrow}|B| \stackrel{d_{B}}{\longrightarrow} \Omega B \longrightarrow 0
$$

is exact.

If $B$ is augmented, then the augmentation $\varepsilon_{B}$ splits the exact sequence, and there is a $K$-module morphism

$$
i_{B}: \Omega B \rightarrow|B|
$$

which splits the exact sequence in the same manner as $\varepsilon_{B}$. We shall call $i_{B}$ the integration of the augmented exact algebra $B$.

An example of an augmented exact algebra $B$ is the algebra of real valued $C^{\infty}$ functions on the real line equipped with the usual differentiation to the module $\Omega B$ of $C^{\infty}$ 1-forms on the real line. The augmentation $\varepsilon_{B}$ is the evaluation map at a 
point $x_{0}$. The integration $i_{B}: \Omega B \rightarrow|B|$ is given by $w \mapsto \int_{x_{0}} w$ and is therefore in conformity with its usual meaning.

5.2. The construction of the algebras $P$ and $Q$ as described in 2.2 and 2.3 can be carried out for an arbitrary augmented algebra $A$. Moreover $P=P(A)$ will be equipped with a derivation and an augmentation. We describe briefly how this is done:

Write $M=\Omega A$ and regard $\mathrm{Sh}(M) \otimes M$ as a Sh $(M)$-module such that $u(v \otimes w)$ $=u \circ v \otimes w, \forall u, v \in \operatorname{Sh}(M)$ and $w \in M$. The linear map Sh $(M) \rightarrow \operatorname{Sh}(M) \otimes M$ given by $1 \mapsto 0$ and $v w \mapsto v \otimes w$ is then a derivation.

Define the ideal $I$ of the shuffle $K$-algebra Sh $(M)$ as in 2.2. The image of $I$ under this derivation is a s!bmodule $I^{*}$ of $\mathrm{Sh}(M) \otimes M$. Define the augmented algebra $P(A)$ such that $|P(A)|=\operatorname{Sh}(M) / I$ and $\Omega P(A)=\operatorname{Sh}(M) \otimes M / I^{*}$. The derivation and the augmentation of $P(A)$ are respectively induced by those of Sh $(M)$.

Denote by $|\rho|: \operatorname{Sh}(M) \rightarrow|P(A)|$ and $\Omega \rho: \operatorname{Sh}(M) \otimes M \rightarrow \Omega P(A)$ the canonical morphisms. Define the morphism of augmented algebras $\chi_{A}: A \rightarrow P(A)$ such that $\left|\chi_{A}\right| a=|\rho|\left(\varepsilon_{A} a+d_{A} a\right)$ and $\Omega \chi_{A} w=\Omega \rho(1 \otimes w), \forall a \in|A|$ and $\forall w \in \Omega A$.

THEOREM. If $B$ is an augmented exact algebra and if $\phi: A \rightarrow B$ is a morphism of augmented algebras, then $\phi$ has a unique factorization

$$
A \stackrel{\chi_{A}}{\longrightarrow} P(A) \longrightarrow B \text {. }
$$

We give a brief outline of a proof of the above theorem as given in [7].

Let $i_{B}: \Omega B \rightarrow|B|$ be the integration of $B$. If $w_{1}^{\prime}, w_{2}^{\prime}, \cdots \in \Omega B$, write $\int w_{1}^{\prime}=i_{B} w_{1}^{\prime}$ and, for $r>1$,

$$
\int w_{1}^{\prime} \cdots w_{r}^{\prime}=i_{B}\left(\left(\int w_{1}^{\prime} \cdots w_{r-1}^{\prime}\right) w_{r}^{\prime}\right)
$$

Define $\phi: P(A) \rightarrow B$ such that, if $u=w_{1} \cdots w_{r} \in \operatorname{Sh}(M)$, then

$$
|\phi||\rho| u=\int\left(\Omega \phi w_{1}\right) \cdots\left(\Omega \phi w_{r}\right) .
$$

Observe that, since $d_{P(A)}$ is surjective, $\Omega \tilde{\phi}$ is determined by $|\tilde{\phi}|$. Verify that $\bar{\phi}$ is the unique morphism of augmented algebras such that $\phi=\phi \chi$.

5.3. Define $Q(A)$ to be the augmented quotient $K$-algebra of $|P(A)|$ over the ideal generated by elements of the type $\left|\chi_{A}\right| a-\eta_{P(A)} \varepsilon_{A} a, \forall a \in|A|$. It turns out that $Q(A)$ can be also taken as a quotient Hopf algebra of the Hopf $K$-algebra Sh $(M)$. The canonical morphism of augmented $K$-algebras $|P(A)| \rightarrow Q(A)$ is universal with respect to morphisms of augmented $K$-algebras $|P(A)| \rightarrow C$, for which the composition $|A| \stackrel{\left|x_{A}\right|}{\longrightarrow}|P(A)| \rightarrow C$ is $\eta_{C} \varepsilon_{A}$.

5.4. In [9], we construct for the Hopf $K$-algebra $Q(A)$ a derivation $\delta$, which has been motivated by calculus of variation. The kernel of the derivation $\delta$ is a Hopf subalgebra $\pi^{1}(A)$ of $Q(A)$. 
In the case of $A$ being the algebra of $C^{\infty}$ functions on the manifold $\mathfrak{M}$, we make the identification $Q=Q(A)$. Then it can be shown that $\pi^{1}(A) \subset \pi^{1}(\mathfrak{M}, p)$. There is a reasonably good possibility that $\pi^{1}(A)=\pi^{1}(\mathfrak{M}, p)$.

The work in [10] can be interpreted as a study of the algebraic aspect of the algebra of iterated path integrals which are independent of paths.

\section{BIBLIOGRAPHY}

1. K. T. Chen, Iterated integrals and exponential homomorphisms, Proc. London Math. Soc. (3) 4 (1954), 502-512. MR 17, 394.

2. - Integration of paths, geometric invariants and a generalized Baker-Hausdorff formula, Ann. of Math. (2) 65 (1957), 163-178. MR 19, 12.

3. - Integration of paths-a faithful representation of paths by non-commutative formal power series, Trans. Amer. Math. Soc. 89 (1958), 395-407. MR 21 \#4992.

4. - Exponential isomorphism for vector spaces and its connection with Lie groups, $\mathrm{J}$. London Math. Soc. 33 (1958), 170-177. MR 21 \#4991.

5. - Formal differential equations, Ann. of Math. (2) 73 (1961), 110-133. MR 27 \#371.

6. - Iterated path integrals and generalized paths, Bull. Amer. Math. Soc. 73 (1967), 935-938. MR 36 \#806.

7. ——, Algebraic paths, J. Algebra 10 (1968), 8-36. MR 37 \#5204.

8. - Homotopy of algebras, J. Algebra 10 (1968), 183-193. MR 38 \#1127.

9. - An algebraic dualization of fundamental groups, Bull. Amer. Math. Soc. 75 (1969), 1020-1024.

10. —, Covering-space-like algebras, J. Algebra 13 (1969), 308-326.

11. _ A sufficient condition for nonabelianness of fundamental groups of differentiable manifolds, Proc. Amer. Math. Soc. 26 (1970), 196-198.

12. H. H. Johnson, A generalization of $K$. T. Chen's invariants for paths under transformation groups, Trans. Amer. Math. Soc. 105 (1962), 453-461. MR 25 \#5125.

13. D. Kraines, Massey higher products, Trans. Amer. Math. Soc. 124 (1966), 431-449. MR 34 \#2010.

14. W. S. Massey, Some higher order cohomology operations, Internat. Sympos. Algebraic Topology, Universidade Nacional Autónoma de México and UNESCO, Mexico City, 1958, pp. 145-154. MR 20 \#4826.

15. J. W. Milnor and J. C. Moore, On the structure of Hopf algebras, Ann. of Math. (2) 81 (1965), 211-264. MR 30 \#4259.

16. A. N. Parsin, A generalization of Jacobian variety, Izv. Akad. Nauk SSSR Ser Mat. 30 (1966), 175-182; English transl., Amer. Math. Soc. Transl. (2) 84 (1969), 187-196. MR 33 \#4956.

17. R. Ree, Lie elements and an algebra associated with shuffles, Ann. of Math. (2) 68 (1958), 210-220. MR 20 \#6447.

18. M. E. Sweedler, Hopf algebras, Benjamin, New York, 1969.

19. A. Weil, Introduction à l'étude des variétés kählériennes, Publ. Inst. Math. Univ. Nancago, VI, Actualités Sci. Indust., no. 1267, Hermann, Paris, 1958. MR 22 \#1921.

UNIVERSITY OF ILLINOIS, URBANA, ILLINOIS 61801 